\title{
rcss: $R$ package for optimal convex stochastic switching
}

by Juri Hinz and Jeremy Yee

\begin{abstract}
The R package rcss provides users with a tool to approximate the value functions in the Bellman recursion under certain assumptions that guarantee desirable convergence properties. This $\mathrm{R}$ package represents the first software implementation of these methods using matrices and nearest neighbors. This package also employs a pathwise dynamic method to gauge the quality of these value function approximations. Statistical analysis can be performed on the results to obtain other useful practical insights. This paper describes rcss version 1.6.
\end{abstract}

\section{Motivation}

Sequential decision making is usually addressed under the framework of discrete-time stochastic control. The theory of Markov Decision Processes/Dynamic Programming provides a variety of methods to deal with such questions. In generic situations, approaching analytical solutions for even some of the simplest decision processes may be a cumbersome process (Powell (2007), Bauerle and Rieder (2011), Pham (2009)). The use of numerical approximations may often be far more practical given the rapid improvements in available computational power. The ability to gauge the quality of these approximations is also of significant importance. This paper will describe the implementation of fast and accurate algorithms to address problems with a finite time setting, finite action set, and convex reward functions whose state processes follow linear dynamics.

Although these assumptions seem restrictive, a large class of practically important control problems can be solved using our methods. For instance, approximating a compact action set by a sufficiently dense finite grid of representative actions, an approximate solution with excellent precision and numerical performance can be obtained. This technique is illustrated by battery storage control (Hinz and Yee (2017a) in the present contribution. Furthermore, various extension to non-linear state dynamics are possible. By using appropriate transformations to linearize the state dynamics, our methodology becomes applicable to control partially observable systems (Hinz and Yee (2017b)).

This $\mathrm{R}$ package implements algorithms to approximate the value functions in finite horizon Markov decision processes and also to gauge the quality of these approximations. Under the conditions discussed in Hinz (2014), these value functions converge to the true unknown value functions uniformly on compact sets. Defined by the maxima half-norms over compact sets, the corresponding topology ensures that the decision rules, inferred from value function approximations, converge pointwise to the optimal decision policy.

This paper points any interested readers to the technical details contained in Hinz (2014). The package rcss represents the first software implementation of these algorithms and has already been used to address problems such as pricing financial options (Hinz and Yap (2015)), natural resource extraction (Hinz et al. (In Press)), battery management (Hinz and Yee (2017a)), and optimal asset allocation under hidden state dynamics (Hinz and Yee (2017b)). One of the major benefits of implementing these methods within R (R Core Team (2013)) is that the results can be analysed using the vast number of statistical tools available in this language.

This R package focuses on linear state dynamics for the continuous state component. While nonlinear dynamics have been covered in Yee (Preprint), linear dynamics allow much of the computational effort to be reduced to a series of multiplications and additions of matrices. This is an attractive feature given the availability of well developed linear algebra libraries. Linear state dynamics also allow for a standardized problem formulation which is desirable for a software package. This $\mathrm{R}$ package also focuses on problems with finite action sets which occur frequently in many real world problems. While this may seem restrictive for other problems, note that action sets are often discretized in practice to allow for numerical tractability.

\section{Problem setting}

Suppose that state space $\mathbf{X}=\mathbf{P} \times \mathbf{Z}$ is the product of a finite set $\mathbf{P}$ and an open convex set $\mathbf{Z} \subseteq \mathbb{R}^{d}$. Furthermore, assume that a finite set $\mathbf{A}$ represents all possible actions. Given a finite time horizon $\{0,1, \ldots, T\} \subset \mathbb{N}$, consider a fully observable controlled Markovian process $\left(X_{t}\right)_{t=0}^{T}:=\left(P_{t}, Z_{t}\right)_{t=0}^{T}$ which consists of two parts. The discrete component $\left(P_{t}\right)_{t=0}^{T}$ describes the evolution of a finitestate controlled Markov chain which takes values in a finite set $\mathbf{P}$. Further assume that at any time 
$t=0, \ldots, T-1$ the controller takes an action $a \in \mathbf{A}$ from a finite set $\mathbf{A}$ of all admissible actions in order to cause the one-step transition from the mode $p \in \mathbf{P}$ to the mode $p^{\prime} \in \mathbf{P}$ with a probability $\alpha_{p, p^{\prime}}(a)$, where $\left(\alpha_{p, p^{\prime}}(a)\right)_{p, p^{\prime} \in \mathbf{P}}$ are pre-specified stochastic matrices for all $a \in \mathbf{A}$.

Let us now turn to the evolution of the other component $\left(Z_{t}\right)_{t=0}^{T}$ of the state process $\left(X_{t}\right)_{t=0}^{T}$. Here, we assume that it follows an uncontrolled evolution in the space $\mathbf{Z}$ driven as

$$
Z_{t+1}=W_{t+1} Z_{t}, \quad t=0, \ldots, T-1
$$

by independent disturbance matrices $\left(W_{t}\right)_{t=1}^{T}$. That is, the transition kernels $\mathcal{K}_{t}^{a}$ governing the evolution of our controlled Markov process $\left(X_{t}\right)_{t=0}^{T}:=\left(P_{t}, Z_{t}\right)_{t=0}^{T}$ from time $t$ to $t+1$ are given for each $a \in \mathbf{A}$ by

$$
\mathcal{K}_{t}^{a} v(p, z)=\sum_{p^{\prime} \in \mathbf{P}} \alpha_{p, p^{\prime}}(a) \mathbb{E}\left(v\left(p^{\prime}, W_{t+1} z\right)\right), \quad p \in \mathbf{P}, z \in \mathbf{Z}, t=0, \ldots, T-1
$$

which acts on each function $v: \mathbf{P} \times \mathbf{Z} \rightarrow \mathbb{R}$ where the above expectations are well-defined.

If the system is in the state $(p, z)$, the costs of applying action $a \in \mathbf{A}$ at time $t=0, \ldots, T-1$ are expressed through $r_{t}(p, z, a)$. Having arrived at time $t=T$ in the state $(p, z)$, a final scrap value $r_{T}(p, z)$ is collected. Thereby the reward and scrap functions

$$
r_{t}: \mathbf{P} \times \mathbf{Z} \times \mathbf{A} \rightarrow \mathbb{R}, \quad r_{T}: \mathbf{P} \times \mathbf{Z} \rightarrow \mathbb{R}
$$

are exogenously given for $t=0, \ldots, T-1$. At each time $t=0, \ldots, T$, the decision rule $\pi_{t}$ is given by a mapping $\pi_{t}: \mathbf{P} \times \mathbf{Z} \rightarrow \mathbf{A}$, prescribing at time $t$ an action $\pi_{t}(p, z) \in \mathbf{A}$ for a given state $(p, z) \in \mathbf{P} \times \mathbf{Z}$. Note that each decision rule refers to the recent state of the system, representing a feedback control. A sequence $\pi=\left(\pi_{t}\right)_{t=0}^{T-1}$ of decision rules is called a policy. For each policy $\pi=\left(\pi_{t}\right)_{t=0}^{T-1}$, the so-called policy value $v_{0}^{\pi}\left(p_{0}, z_{0}\right)$ is defined as the total expected reward

$$
v_{0}^{\pi}\left(p_{0}, z_{0}\right)=\mathbb{E}^{\left(p_{0}, z_{0}\right), \pi}\left[\sum_{t=0}^{T} r_{t}\left(P_{t}, Z_{t}, \pi_{t}\left(P_{t}, Z_{t}\right)\right)+r_{t}\left(P_{t}, Z_{t}\right)\right] .
$$

In this formula $\mathbb{E}^{\left(p_{0}, z_{0}\right), \pi}$ stands for the expectation with respect to the probability distribution of $\left(X_{t}\right)_{t=0}^{T}:=\left(P_{t}, Z_{t}\right)_{t=0}^{T}$ defined by Markov transitions from $\left(P_{t}, Z_{t}\right)$ to $\left(P_{t+1}, Z_{t+1}\right)$, which are induced by the kernels $\mathcal{K}_{t}^{\pi_{t}\left(P_{t}, Z_{t}\right)}$ for $t=0, \ldots, T-1$, started at the initial point $\left(P_{0}, Z_{0}\right)=\left(p_{0}, z_{0}\right)$.

Now we turn to the optimization goal. A policy $\pi^{*}=\left(\pi_{t}^{*}\right)_{t=0}^{T-1}$ is called optimal if it maximizes the total expected reward over all policies $\pi \mapsto v_{0}^{\pi}(p, z)$. To obtain such policy, one introduces for $t=0, \ldots, T-1$ the so-called Bellman operator

$$
\mathcal{T}_{t} v(p, z)=\max _{a \in \mathbf{A}}\left[r_{t}(p, z, a)+\sum_{p^{\prime} \in \mathbf{P}} \alpha_{p, p^{\prime}}(a) \mathbb{E}\left[v\left(p^{\prime}, W_{t+1} z\right)\right]\right]
$$

for $(p, z) \in \mathbf{P} \times \mathbf{Z}$, acting on all functions $v$ where the stochastic kernel is defined. Consider the Bellman recursion, also referred to as backward induction:

$$
v_{T}=r_{T}, \quad v_{t}=\mathcal{T}_{t} v_{t+1} \quad \text { for } t=T-1, \ldots, 0 .
$$

Having assumed that the reward functions are convex and globally Lipschitz and the disturbances $W_{t+1}$ are integrable, there exists a unique recursive solution $\left(v_{t}^{*}\right)_{t=0}^{T}$ to the Bellman recursion. These functions $\left(v_{t}^{*}\right)_{t=0}^{T}$ are called value functions and they determine an optimal policy (possibly not unique) $\pi^{*}=\left(\pi_{t}^{*}\right)_{t=0}^{T}$ via

$$
\pi_{t}^{*}(p, z)=\arg \max _{a \in \mathbf{A}}\left[r_{t}(p, z, a)+\sum_{p^{\prime} \in \mathbf{P}} \alpha_{p, p^{\prime}}(a) \mathbb{E}\left[v_{t+1}^{*}\left(p^{\prime}, W_{t+1} z\right)\right],\right.
$$

for $t=T-1, \ldots, 0$.

The aim of the package rcss is to approximate the true value functions $\left(v_{t}^{*}\right)_{t=0}^{T-1}$ and the corresponding optimal policies $\pi^{*}=\left(\pi_{t}^{*}\right)_{t=0}^{T-1}$. Given these approximations, our package also determines their distance to optimality which will allow the user to decide whether it is within some acceptable margin or whether further fine tuning is needed to obtain even more accurate results. 


\section{Numerical approach}

Since the reward and scrap functions are convex in the continuous variable, the value functions are also convex and can be approximated by piecewise linear and convex functions. For this, introduce the so-called subgradient envelope $\mathcal{S}_{\mathbf{G}^{m}} f$ of a convex function $f: \mathbf{Z} \rightarrow \mathbb{R}$ on a grid $\mathbf{G}^{m} \subset \mathbf{Z}$ with $m$ points:

$$
\left(\mathcal{S}_{\mathbf{G}^{m}} f\right)(z)=\max _{g \in \mathbf{G}^{m}}\left(\nabla_{g} f\right)(z),
$$

for $z \in \mathbf{Z}$ where $\nabla_{g} f$ is the tangent of $f$ at grid point $g \in \mathbf{G}^{m}$. Using the subgradient envelope operator, define the double-modified Bellman operator as

$$
\mathcal{T}_{t}^{m, n} v(p, z)=\mathcal{S}_{\mathbf{G}^{m}} \max _{a \in \mathbf{A}}\left(r_{t}(p, z, a)+\sum_{p^{\prime} \in \mathbf{P}} \alpha_{p, p^{\prime}}^{a} \sum_{k=1}^{n} v_{t+1}^{(k)} v\left(p^{\prime}, W_{t+1}^{(k)} z\right)\right),
$$

where the probability weights $\left(v_{t+1}^{(k)}\right)_{k=1}^{n}$ correspond to the distribution sampling $\left(W_{t+1}^{(k)}\right)_{k=1}^{n}$ of each disturbance $W_{t+1}$. The corresponding backward induction

$$
\begin{aligned}
v_{T}^{m, n}(p, z) & =\mathcal{S}_{\mathbf{G}^{m}} r_{T}(p, z), \\
v_{t}^{m, n}(p, z) & =\mathcal{T}_{t}^{m, n} v_{t+1}^{m, n}(p, z), \quad t=T-1, \ldots 0,
\end{aligned}
$$

yields the so-called double-modified value functions $\left(v_{t}^{m, n}\right)_{t=0}^{T}$. Under appropriate assumptions regarding grid density and the disturbance sampling, the double-modified value functions converge uniformly to the true value functions on compact sets (see Hinz (2014)). To gauge the quality of the above approximations, we construct two random variables whose expectations bound the true value function i.e.

$$
\mathbb{E}\left(\underline{v}_{0}\left(p, z_{0}\right)\right) \leq v_{0}\left(p, z_{0}\right) \leq \mathbb{E}\left(\bar{v}_{0}\left(p, z_{0}\right)\right), \quad p_{0} \in \mathbf{P}, \quad z_{0} \in \mathbf{Z} .
$$

We refer any interested readers to Hinz and Yap (2015) for the technical details. This process exhibits a helpful self-tuning property. The closer the value function approximations resemble their true unknown counterparts, the tighter the bounds in Equation 6 and the lower the standard errors of the bound estimates. The R package rcss represents these piece-wise linear functions as matrices and utilises nearest neighbor algorithms (from Mangenat and Jefferies (2018)) to reduce the computational effort. Most of the computational work is done in C++ via Rcpp (Eddelbuettel and Francois (2011)) and is parallelized using OpenMp (Dagum and Menon (1998)). The following sections give some code demonstrations.

\section{Example: Bermuda put}

Stochastic control problems of optimal switching type naturally arise in the framework of valuation of financial contracts. A simple example is given by the Bermudan put option. This option gives its owner the right but not an obligation to choose a time to exercise the option in a finite number of time points before the maturity of the option in order to receive a payment which depends on the price of the underlying asset at the exercise time. The so-called fair price of the Bermudan option is related to the solution of an optimal stopping problem (see Glasserman (2003)). Here, the risk-neutral undiscounted asset price process $\left(\tilde{Z}_{t}\right)_{t=0}^{T}$ at time steps $0, \ldots, T$ is modeled as a sampled geometric Brownian motion

$$
\tilde{Z}_{t+1}=\epsilon_{t+1} \tilde{Z}_{t}, \quad t=0, \ldots, T-1, Z_{0} \in \mathbb{R}_{+},
$$

where $\left(\varepsilon_{t}\right)_{t=1}^{T}$ are independent identically distributed random variables following a log-normal distribution, usually modeled in terms of

$$
\varepsilon_{t}=\exp \left(\left(\left(\mu-\frac{\sigma^{2}}{2}\right) \Delta+\sigma \sqrt{\Delta} N_{t}\right),\right.
$$

with independent, identically standard normally distributed random variables $\left(N_{t}\right)_{t=1}^{T}$ where the parameters $\sigma>0$ and $\mu \in \mathbb{R}_{+}$represent the stock price volatility and the continuously compounded interest rate respectively, measured on yearly scale. The tenor $\Delta$ describes the length (measured in years) of the equidistant time interval between exercise times. The fair price of such option with strike price $K$, interest rate parameter $\rho=\mu \Delta$, and maturity date $\Delta T$, is given by the solution to the optimal stopping problem

$$
\sup \left\{\mathbb{E}\left(e^{-\rho \tau} \max \left(\left(K-\tilde{Z}_{\tau}\right), 0\right)\right): \quad \tau \text { is }\{0,1, \ldots, T\} \text {-valued stopping time }\right\} .
$$


A transformation of the state space is required to represent the reward functions in a convenient way. Thus, we introduce an augmentation with 1 via

$$
Z_{t}=\left[\begin{array}{c}
1 \\
\tilde{Z}_{t}
\end{array}\right], \quad t=0, \ldots, T
$$

then it becomes possible to represent the evolution as the linear state dynamics

$$
Z_{t+1}=W_{t+1} Z_{t}, \quad t=0, \ldots, T-1
$$

with independent and identically distributed matrix-valued random variables $\left(W_{t}\right)_{t=1}^{T}$ given by

$$
W_{t+1}=\left[\begin{array}{cc}
1 & 0 \\
0 & \epsilon_{t+1}
\end{array}\right], \quad t=0, \ldots, T-1 .
$$

This switching system is defined by two positions $\mathbf{P}=\{1,2\}$ and two actions $\mathbf{A}=\{1,2\}$. Here, the positions "exercised" and "not exercised" are represented by $p=1$ and $p=2$ respectively, and the actions "don't exercise" and "exercise" are denoted by $a=1$ and $a=2$ respectively. With this interpretation, the position change is given by deterministic transitions to specified states:

$$
\alpha_{p, p^{\prime}}^{a}= \begin{cases}1 & \text { if } p^{\prime}=\alpha(p, a) \\ 0 & \text { else }\end{cases}
$$

deterministically determined by the target positions

$$
(\alpha(p, a))_{p, a=1}^{2} \sim\left[\begin{array}{ll}
\alpha(1,1) & \alpha(1,2) \\
\alpha(2,1) & \alpha(2,2)
\end{array}\right] .=\left[\begin{array}{ll}
1 & 1 \\
2 & 1
\end{array}\right],
$$

While the rewards at time $t=0, \ldots, T$, are defined as

$$
\begin{array}{r}
r_{t}\left(p,\left(z^{(1)}, z^{(2)}\right), a\right)=e^{-\rho t} \max \left(K-z^{(2)}, 0\right)(p-\alpha(p, a)), \\
r_{T}\left(p,\left(z^{(1)}, z^{(2)}\right)\right)=e^{-\rho T} \max \left(K-z^{(2)}, 0\right)(p-\alpha(p, 2)),
\end{array}
$$

for all $p \in \mathbf{P}, a \in \mathbf{A}, z \in \mathbb{R}_{+}$.

\section{Code example}

As a demonstration, let us consider a Bermuda put option with strike price 40 that expires in 1 year. The put option is exercisable at 51 evenly spaced time points in the year, which includes the start and end of the year. The following code approximates the value functions in the Bellman recursion.

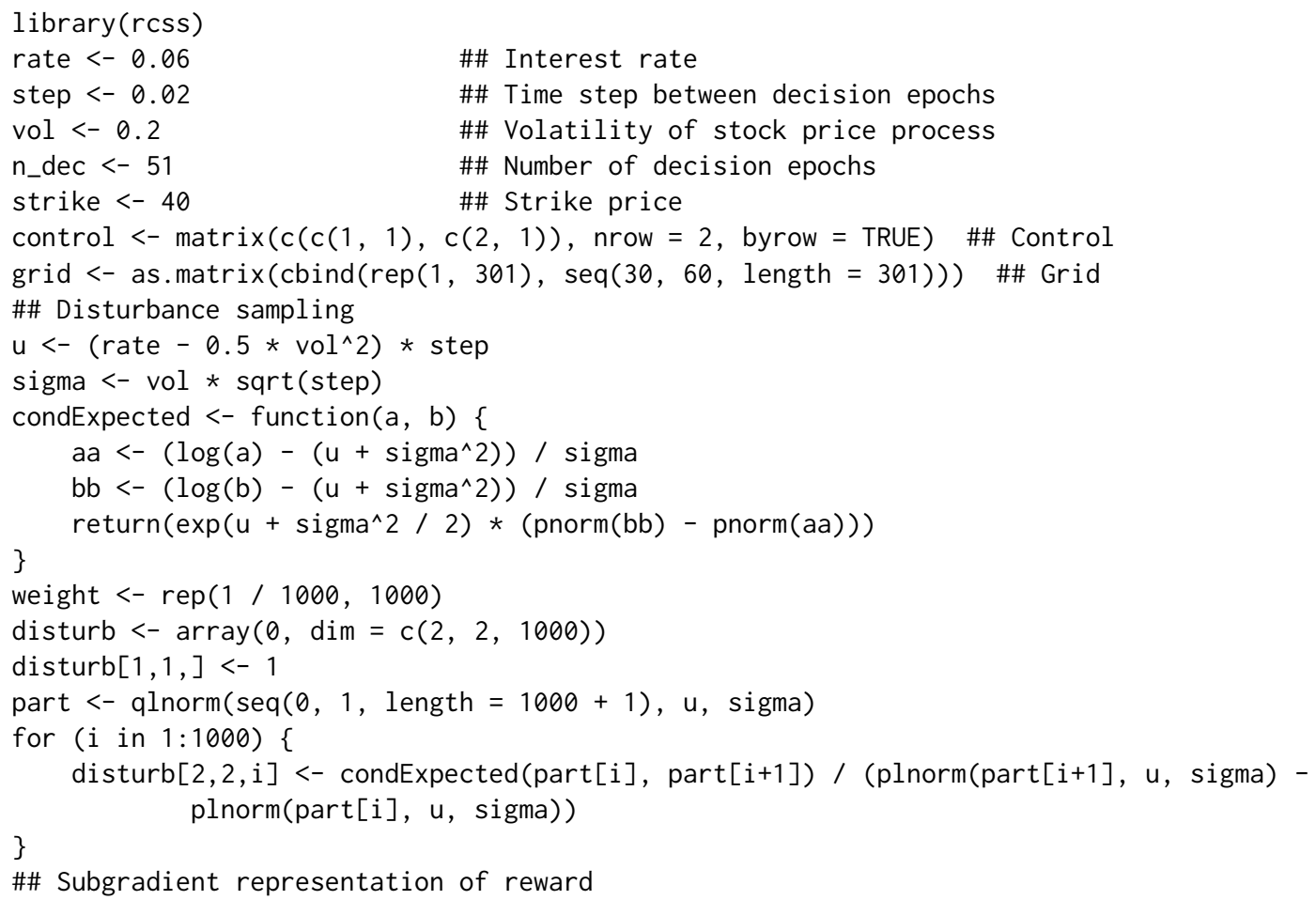




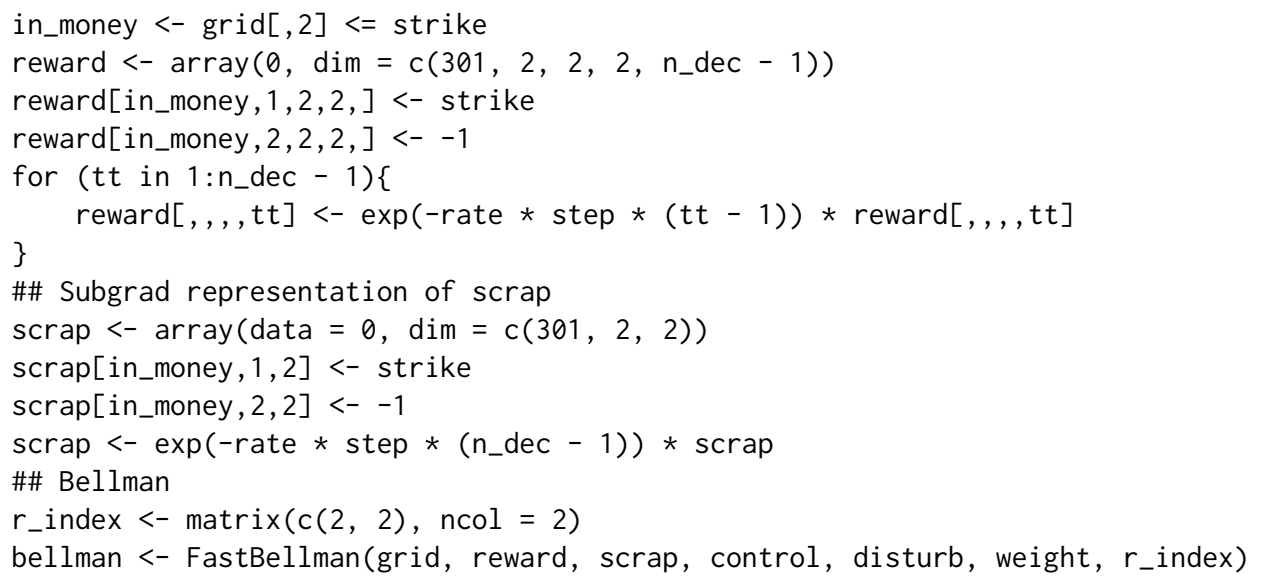

The matrix grid represents the grid points where each row represents a point. The 3-dimensional array disturb represents the sampling of the disturbances where disturb $[,, i]$ gives the i-th sample. Here, we use local averages on a 1000 component partition of the disturbance space. The 5-dimensional array reward represents the subgradient approximation with reward[, , a, $p, t]$ representing $\mathcal{S}_{\mathrm{G}^{m}} r_{t}(p, ., a)$. The object bellman is a list containing the approximations of the value functions and expected value functions for all positions and decision epochs. Please refer to the package manual for the format of the inputs and outputs. To visualise the value function of the Bermuda put option, simply run the following plot command:

$\operatorname{plot}(\operatorname{grid}[, 2], \operatorname{rowSums}(\operatorname{bellman} \$ \operatorname{value}[,, 2,1] *$ grid), type $=" 1 ", x l a b=$ "Stock Price", $\mathrm{ylab}=$ "Option Value")

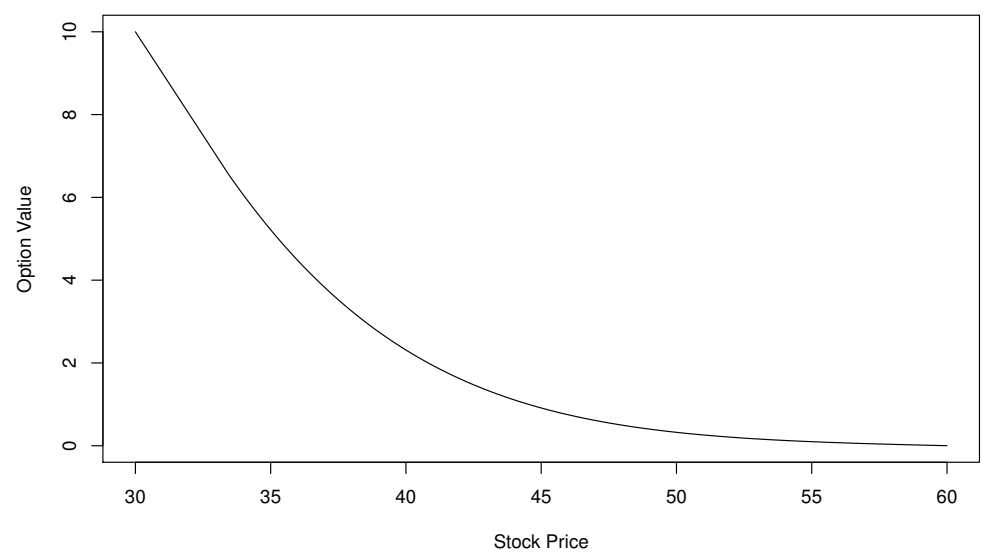

Figure 1: Bermuda put value function.

The following code then performs the solution diagnostics by computing the lower and upper bound estimates for the value of the option when $Z_{0}=36$.

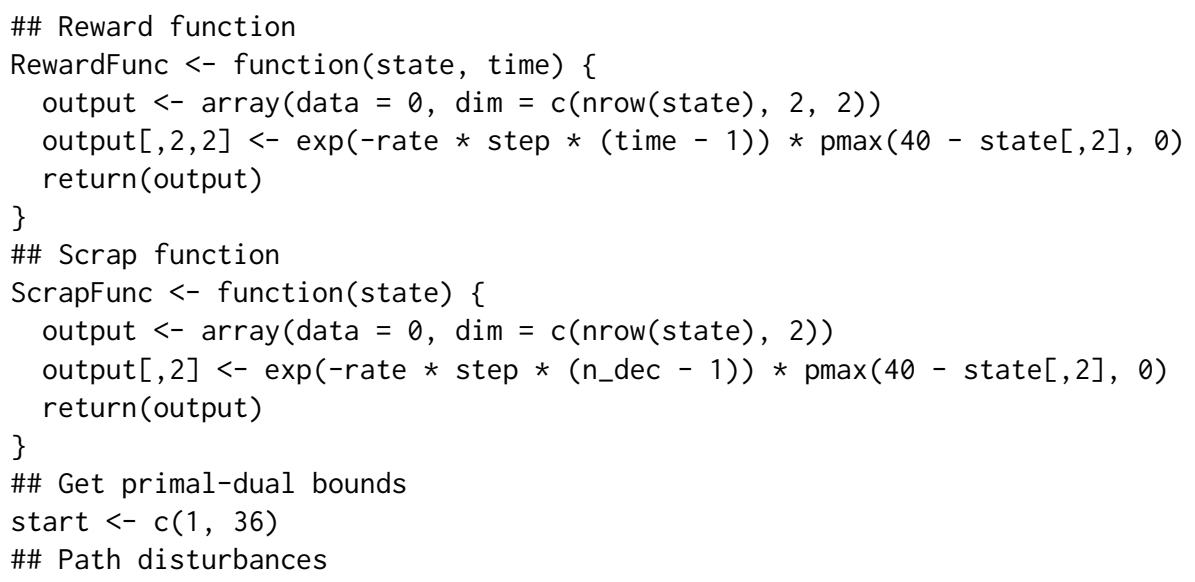


set. seed (12345)

n_path $<-500$

path_disturb $<-\operatorname{array}(0, \operatorname{dim}=c(2,2$, n_path, n_dec -1$))$

path_disturb[1, $1,,]<-1$

rand1 <- rnorm(n_path * (n_dec - 1) / 2)

rand $1<-$ as. vector ( $r$ bind (rand1, -rand1))

path_disturb $[2,2,]<,-\exp \left(\left(\right.\right.$ rate $\left.-0.5 * \operatorname{vol}{ }^{\wedge} 2\right) * \operatorname{step}+\operatorname{vol} * \operatorname{sqrt}($ step $) *$ rand 1$)$

path <- PathDisturb(start, path_disturb)

policy <- FastPathPolicy (path, grid, control, RewardFunc, bellman\$expected)

\#\# Subsim disturbances

n_subsim $<-500$

subsim <- $\operatorname{array}(0, \operatorname{dim}=c(2,2$, n_subsim, n_path, $($ n_dec -1$)))$

subsim $[1,1,,]<$,

rand2 <- rnorm(n_subsim * n_path * (n_dec - 1) / 2)

rand2 <- as. vector ( $r$ bind ( $r$ and2, - rand2))

subsim[2,2, , ] <- $\exp \left(\left(\right.\right.$ rate $\left.-0.5 * \operatorname{vol}{ }^{\wedge} 2\right) * \operatorname{step}+\operatorname{vol} * \operatorname{sqrt}($ step $) *$ rand 2$)$

subsim_weight <- $\operatorname{rep}(1 /$ n_subsim, n_subsim)

mart <- FastAddDual (path, subsim, subsim_weight, grid, bellman\$value, ScrapFunc)

bounds <- AddDualBounds(path, control, RewardFunc, ScrapFunc, mart, policy)

The above code takes the exact reward and scrap functions as inputs. The function FastPathPolicy computes the candidate optimal policy. The object bounds is a list containing the primals and duals for each sample path $i$ and each position $p$ at each decision time $t$. Again, please refer to the package manual for the format of the inputs and outputs. If the price of the underlying asset is 36, the $99 \%$ confidence interval for the option price is given by the following.

$>\operatorname{print}($ GetBounds(bounds, $0.01,2$ ))

[1] 4.4758024 .480533

The package rcss also allows the user to test the prescribed policy from the Bellman recursion on any supplied set of sample paths. The resulting output can then be further studied with time series analysis or other statistical work. In the following code, we will use the previously generated 500 sample paths to backtest our policy and generate histograms.
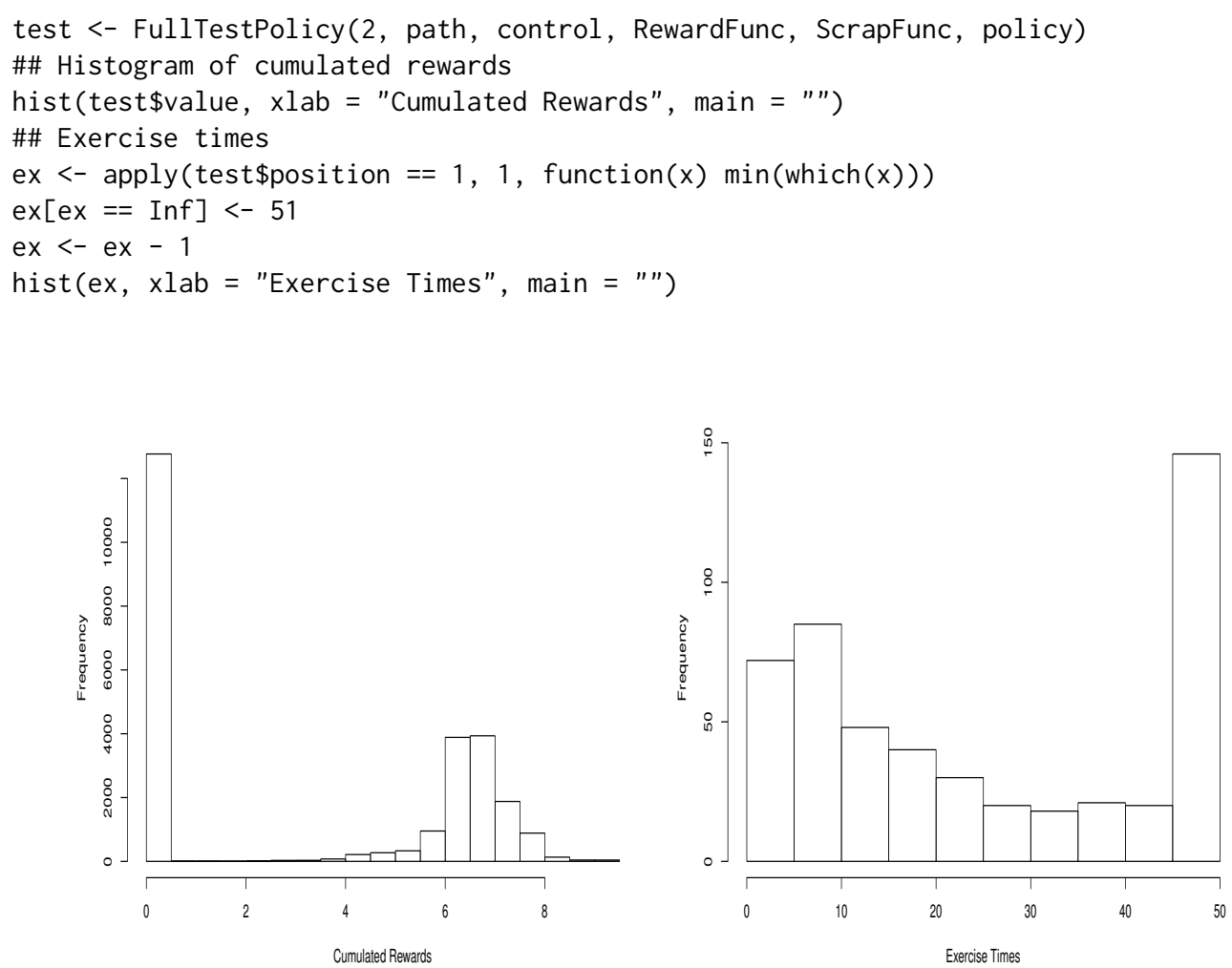

Figure 2: Distribution of cumulated rewards and exercise times.

Figure 2 contains the histograms for the cumulated rewards and exercise times. These can be useful to a practitioner (e.g., anticipating when the holder will exercise their option). Let us emphasise 
the usefulness of such scenario generation. Given an approximately optimal policy and backtesting, one can perform statistical analysis on the backtested values to obtain practical insights such as for risk analysis purposes.

\section{Example: swing option}

Let us now consider the swing option which is a financial contract popular in the energy business. In the simplest form, it gives the owner the right to obtain a certain commodity (such as gas or electricity) at a pre-specified price and volume at a number of exercise times which can be freely chosen by the contract owner. Let us consider a specific case of such a contract, referred to as a unit-time refraction period swing option. In this contract, there is a limit to exercise only one right at any time. Given the discounted commodity price $\left(S_{t}\right)_{t=0}^{T}$, the so-called fair price of a swing option with $N$ rights is given by the supremum,

$$
\sup _{0 \leq \tau_{1}<\cdots<\tau_{N} \leq T} \mathbb{E}\left[\sum_{n=1}^{N}\left(S_{\tau_{n}}-K e^{-\rho \tau_{n}}\right)^{+}\right],
$$

over all stopping times, $\tau_{1}, \ldots, \tau_{N}$, with values in $\{0, \ldots, T\}$. In order to represent this control problem as a switching system, we use the position set $\mathbf{P}=\{1, \ldots, N+1\}$ to describe the number of exercise rights remaining. That is, $p \in \mathbf{P}$ stands for the situation when there are $p-1$ rights remaining to be exercised. The action set $\mathbf{A}=\{1,2\}$ represents the choice between exercising $(a=1)$ or not exercising $(a=2)$. The control matrices $\left(\alpha_{p, p^{\prime}}^{a}\right)$ are given for exercise action $a=1$

$$
\alpha_{p, p^{\prime}}^{1}= \begin{cases}1 & \text { if } p^{\prime}=\max \{1,(p-1)\} \\ 0 & \text { else }\end{cases}
$$

and for not-exercise action $a=2$ as

$$
\alpha_{p, p^{\prime}}^{2}= \begin{cases}1 & \text { if } p^{\prime}=p \\ 0 & \text { else }\end{cases}
$$

for all $p, p^{\prime} \in \mathbf{P}$. In the case of the swing option, the transition between $p$ and $p^{\prime}$ occurs deterministically, since once the controller decides to exercise the right, the number of rights remaining is diminished by one. The deterministic control of the discrete component is easier to describe in terms of the matrix $(\alpha(p, a))_{p \in \mathbf{P}, a \in \mathbf{A}}$ where $p^{\prime}=\alpha(p, a) \in \mathbf{P}$ stands for the discrete component which is reached from $p \in \mathbf{P}$ by the action $a \in \mathbf{A}$. For the case of the swing option this matrix is

$$
(\alpha(p, a))_{p \in \mathbf{P}, a \in \mathbf{A}}=\left[\begin{array}{cc}
1 & 1 \\
1 & 2 \\
2 & 3 \\
\cdots & \cdots \\
N & N+1
\end{array}\right] .
$$

Having modelled the discounted commodity price process as an exponential mean-reverting process with a reversion parameter $\kappa \in[0,1[$, long run mean $\mu>0$, and volatility $\sigma>0$, we obtain the logarithm of the discounted price process as

$$
\tilde{Z}_{t+1}=(1-\kappa)\left(\tilde{Z}_{t}-\mu\right)+\mu+\sigma \epsilon_{t+1}, \quad \tilde{Z}_{0}=\ln \left(S_{0}\right) .
$$

A further transformation of the state space is required before linear state dynamics can be achieved. If we introduce an augmentation with 1 via

$$
Z_{t}=\left[\begin{array}{c}
1 \\
\tilde{Z}_{t}
\end{array}\right], \quad t=0, \ldots, T
$$

then it becomes possible to represent the evolution as the linear state dynamics

$$
Z_{t+1}=W_{t+1} Z_{t}, \quad t=0, \ldots, T-1,
$$

with independent and identically distributed matrix-valued random variables $\left(W_{t}\right)_{t=1}^{T}$ given by

$$
W_{t+1}=\left[\begin{array}{cc}
1 & 0 \\
\kappa \mu+\sigma \epsilon_{t+1} & (1-\kappa)
\end{array}\right], \quad t=0, \ldots, T-1 .
$$

The reward and scrap values are given by

$$
r_{t}\left(p,\left(z^{(1)}, z^{(2)}\right), a\right)=\left(e^{z^{(2)}}-K e^{-\rho t}\right)^{+}(p-\alpha(p, a))
$$


for $t=0, \ldots, T-1$ and

$$
r_{T}\left(p,\left(z^{(1)}, z^{(2)}\right)\right)=\left(e^{z^{(2)}}-K e^{-\rho T}\right)^{+}(p-\alpha(p, 1))
$$

respectively for all $p \in \mathbf{P}$ and $a \in \mathbf{A}$.

\section{Code example}

In this example, consider a swing option with 5 rights exercisable on 101 time points. As before, we begin by performing the value function approximation.

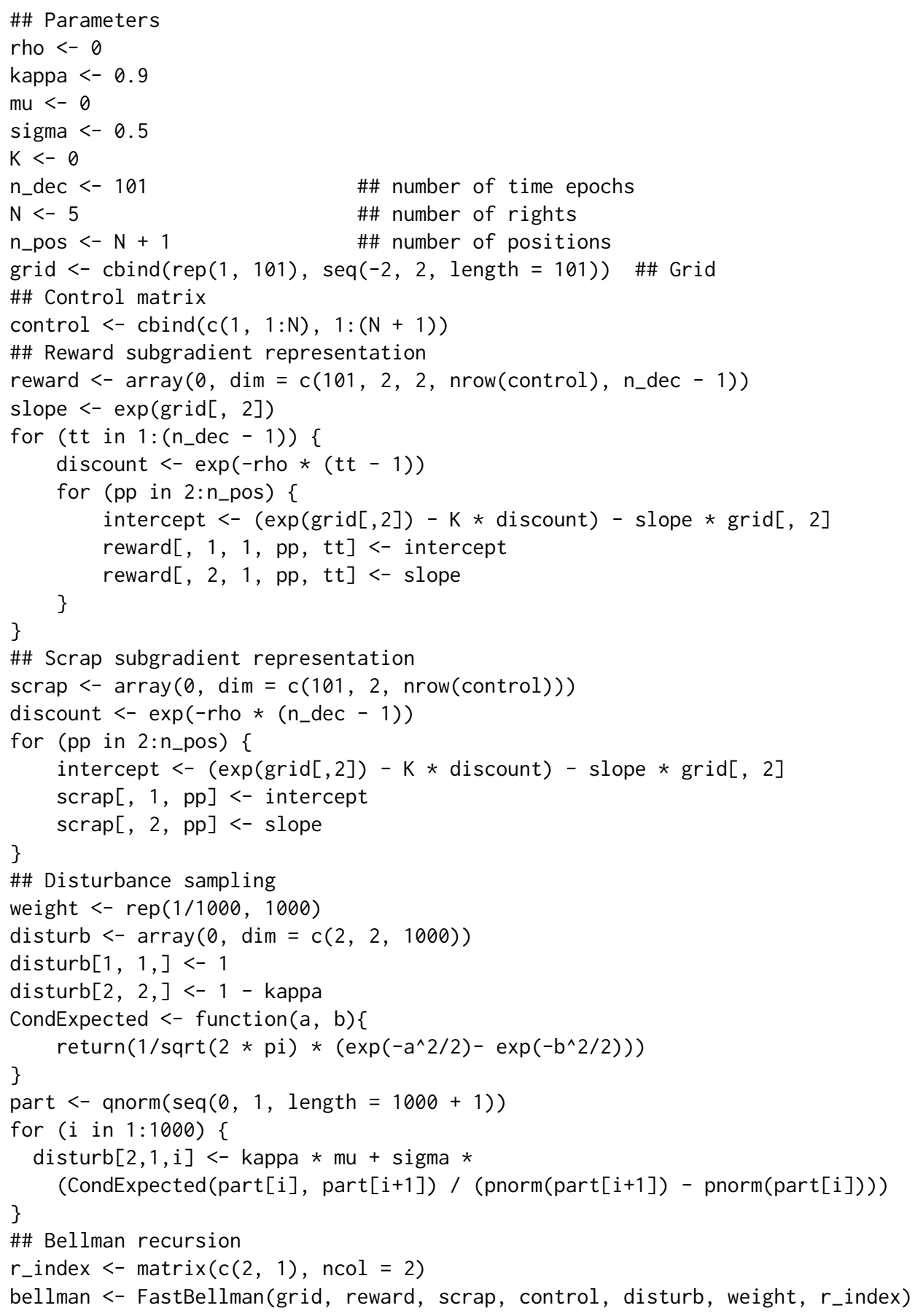

After obtaining these function approximations, the following code computes the $99 \%$ confidence intervals for the value of a swing option with 5 remaining rights.

\#\# Exact reward function

RewardFunc <- function(state, time) \{

output $<-\operatorname{array}(\theta, \operatorname{dim}=c($ nrow $($ state $), 2, \operatorname{nrow}($ control $)))$ 


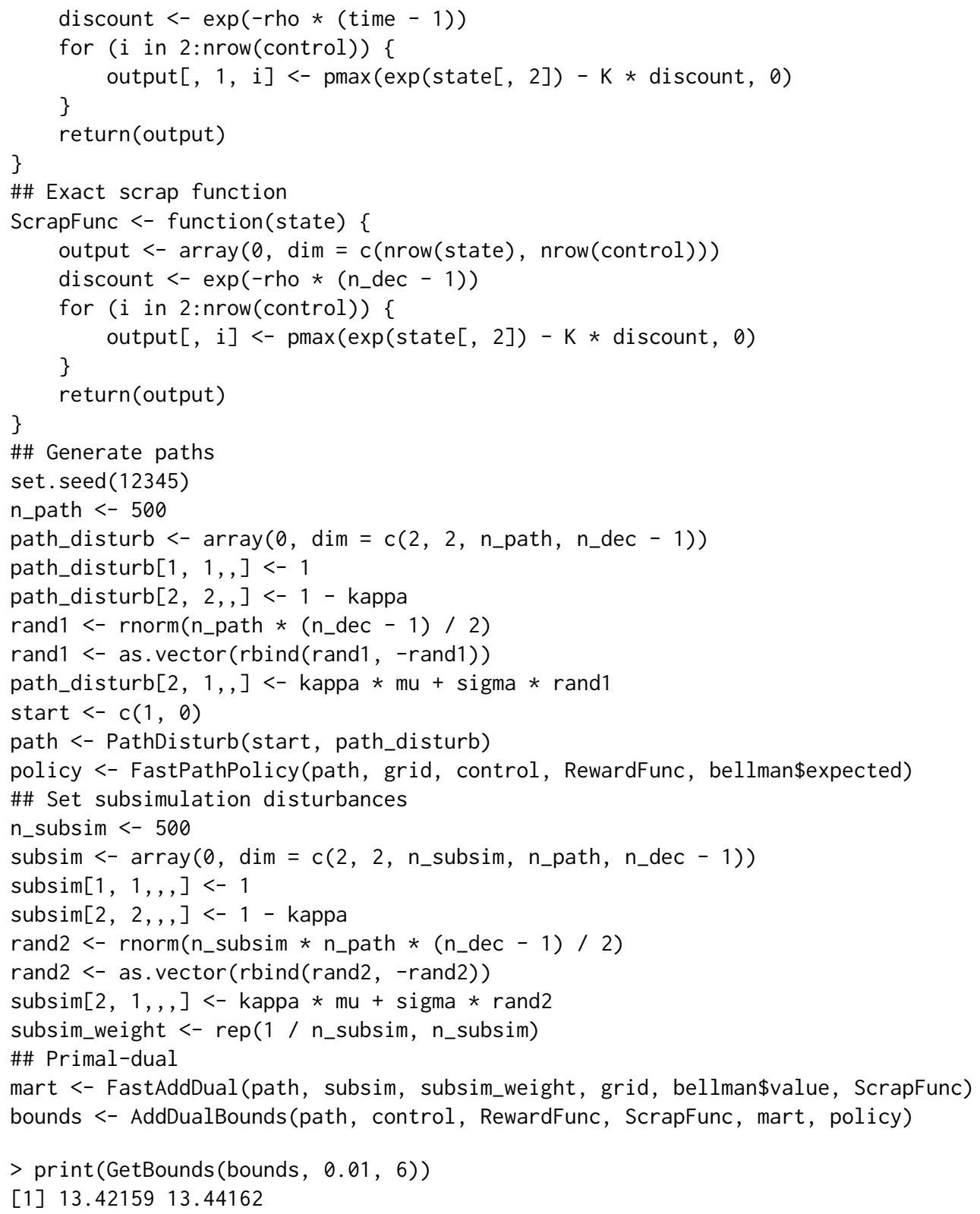

The tight $99 \%$ confidence interval for the price of the swing option above seems to indicate that the function approximations are of a good quality. With this in mind, we now proceed to backtesting the associated policy. We reuse the 1,000 sample path generated for the solution diagnostics.

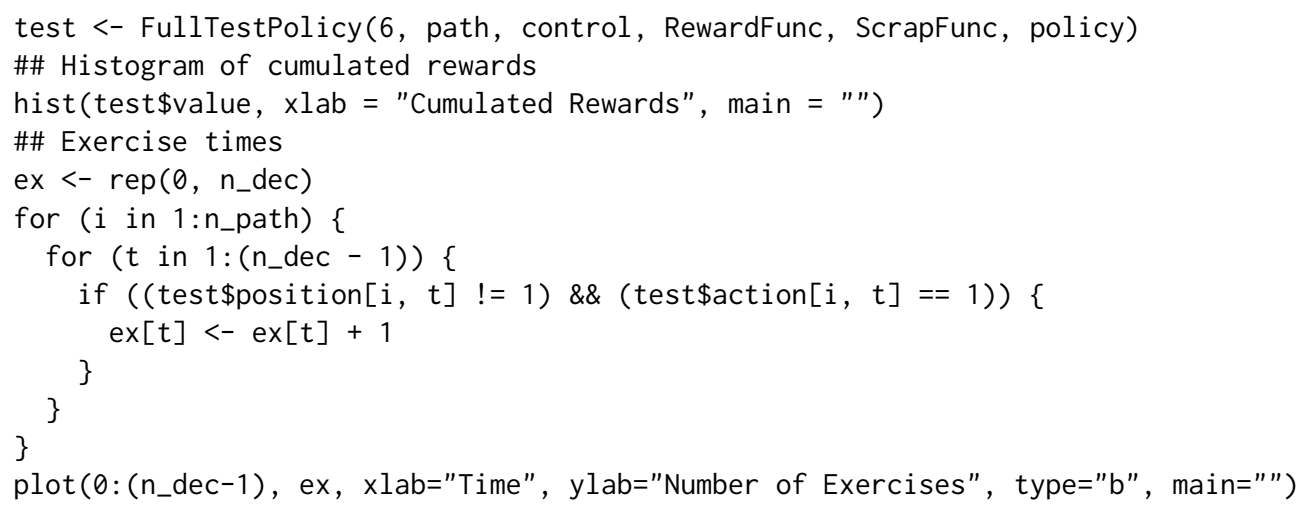

Figure 3 show the histogram for the cumulated rewards and the number of exercises at each time point over the 1,000 sample paths. As mentioned before, more sophisticated statistical analysis can be performed on these path values to gain practical insights. 

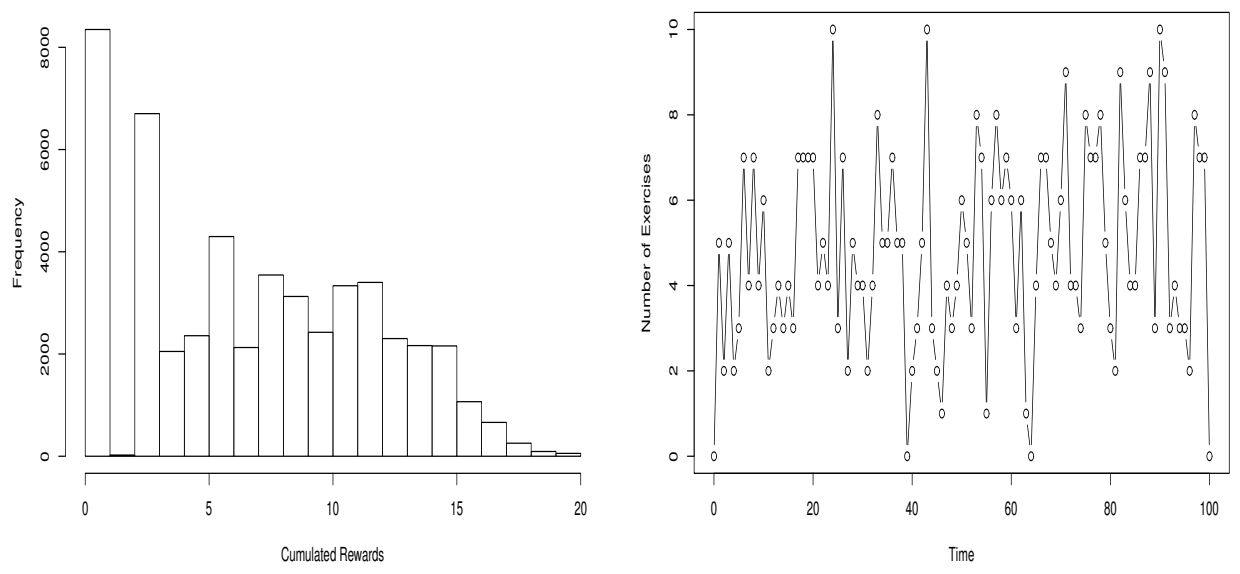

Figure 3: Distribution of cumulated rewards and number of exercises.

\section{Example: optimal energy storage}

This section examines optimal energy storage for an energy retailer in the presence of a battery. The model is beyond the scope of this paper and this paper points any interested readers to Hinz and Yee (2017a) for more details.

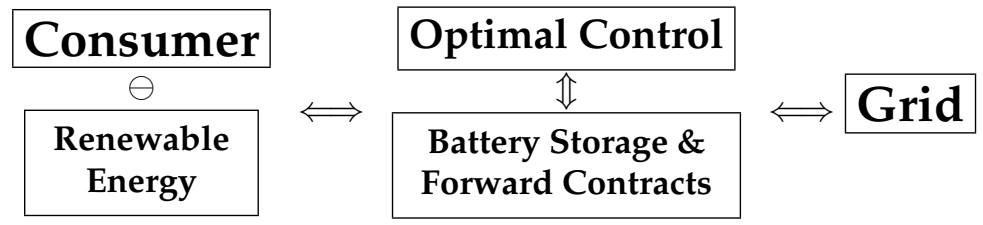

Figure 4: Energy dispatch in the presence of renewable energy and battery storage.

The model is represented by the above figure and the basic gist is as follows:

- At every decision epoch, the energy retailer faces an energy imbalance determined by the net energy demand and the retailer's forward position in the electricity market.

- Any real time imbalance must be rectified by buying or selling at real time grid prices.

- When the electricity retailer has access to a battery, any imbalances can be offset (at least partially) by stored energy. This battery has fixed capacity.

- The retailer's actions is given by the size of the retailer's forward positions in the electricity market. These actions determine the expected amount of energy stored in the battery at each time.

- The goal of the retailer is to reduce the amount of balancing costs and forward trading costs.

The code below begins by specifying the grid, the battery specifications, the action set, and the effect of the action on the battery level. The grid will be used to represent the continuous state component that drives the forward price in the electricity market. Here, the battery has capacity 100 and discretized into steps of 5 from 0 . The action set represents the safety margin (the amount of energy bought in the day ahead market that exceeds the predicted net demand) that the retailer may choose. The control array below captures the transition probabilities of the expected change in the battery level based on the current battery level and the retailer's safety margin.

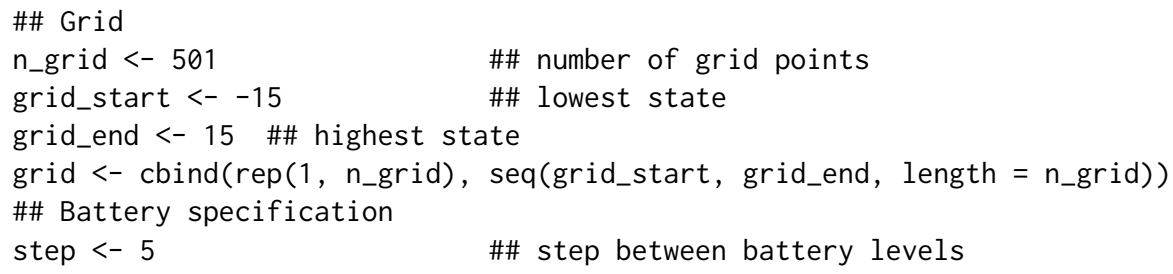




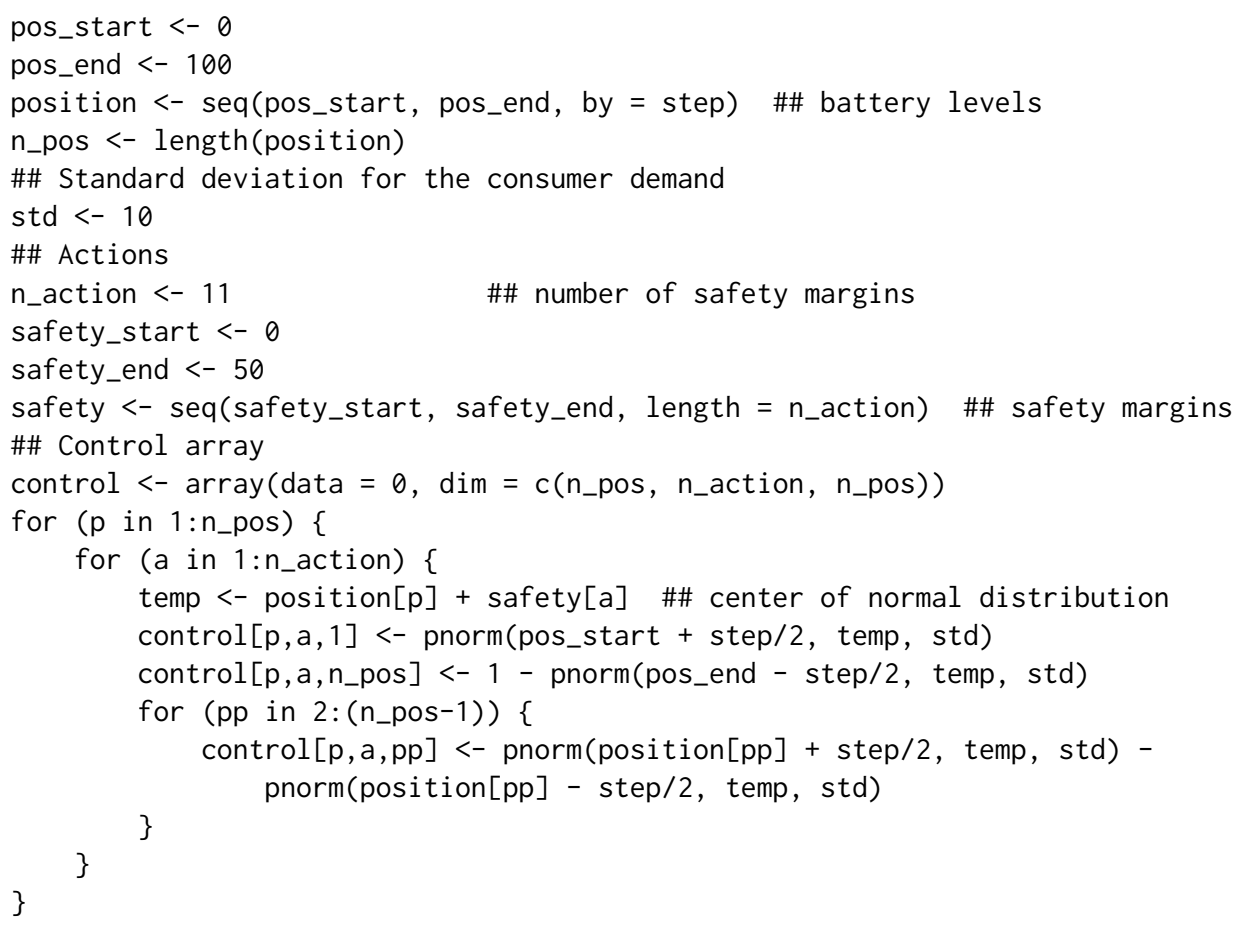

Next the code computes the expected excess and shortages of electricity faced by the retailer at each time period.

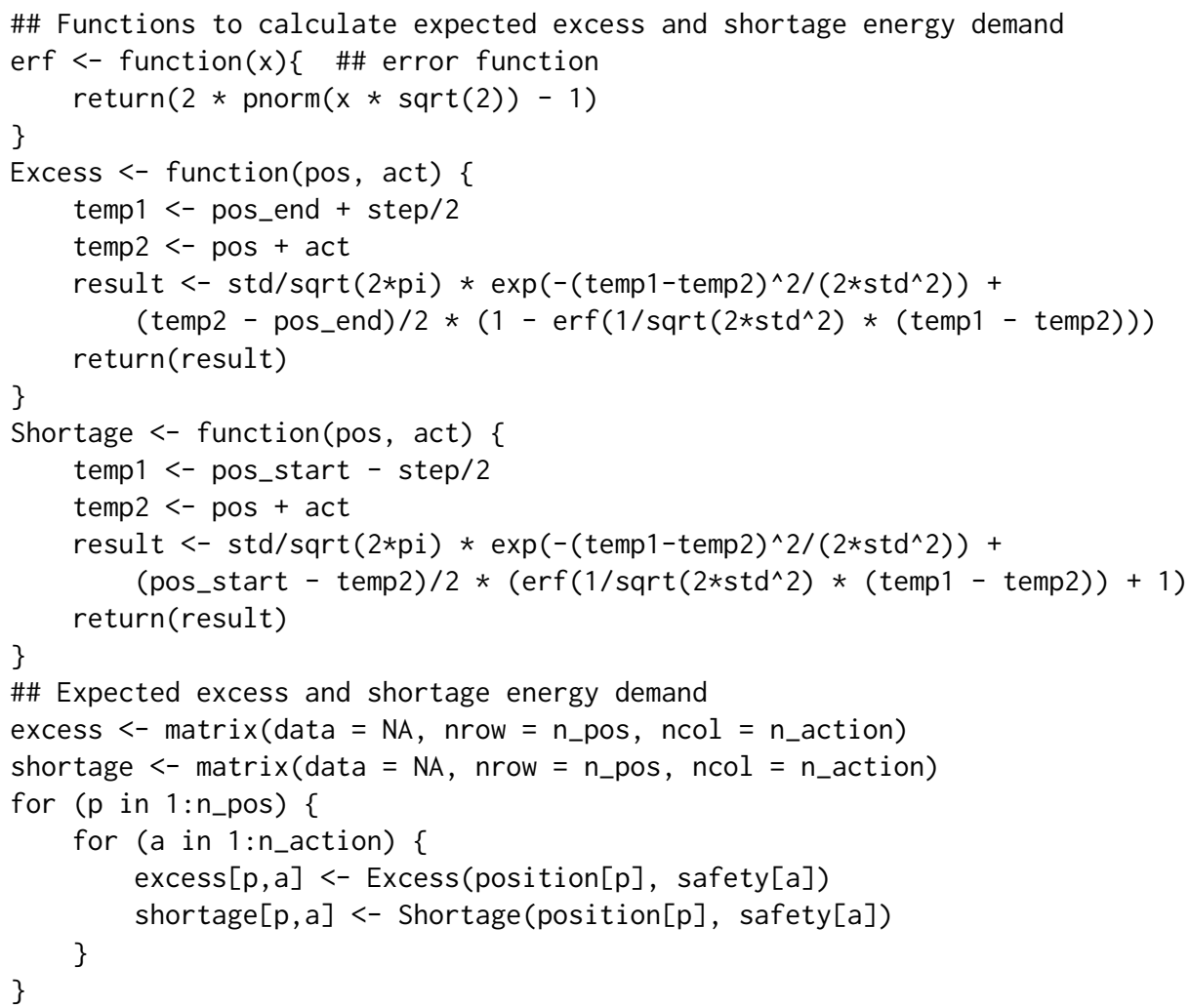

The subgradient envelope of the reward and scrap functions are then specified below. Essentially, the reward is given by the cost of the forward trading and the cost of any expected shortages or excess energy that needs to be balanced. At the final time, the retailer sells all stored energy in the battery at the forward price. This is captured by the scrap reward function.

\#\# Subgradient representation of reward functions

n_dec $<-48 * 7$ \#\# number of decision epochs

$u_{-} t<-10+\cos \left(\left(\theta:\left(n_{-}\right.\right.\right.$dec -1$\left.)\right) * 2 *$ pi $/ 48+3 *$ pi $\left./ 2\right)$

$v_{-} t<-1+\left(\sin \left(\left(\theta:\left(n_{-}\right.\right.\right.\right.$dec -1$\left.\left.\left.)\right) * 2 * p i / 48+3 * p i / 2\right)\right) / 2$ 


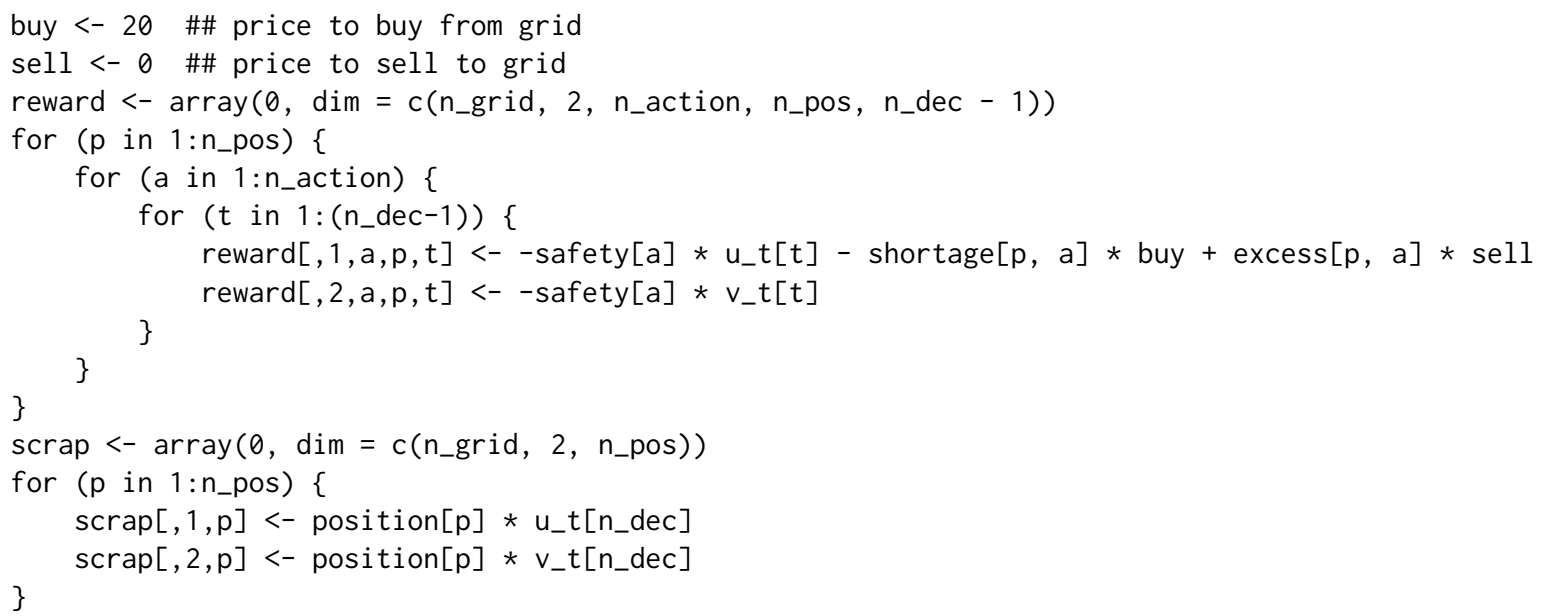

Finally, the disturbance sampling and the resulting double modified value functions are computed using the subgradient approach. Here, we assume that the continuous state $\left(Z_{t}\right)$ driving the forward price is an autoregression of order one. For the disturbance sample, we partition the disturbance space into 1000 components of equal probability measure and take the local average on each component.

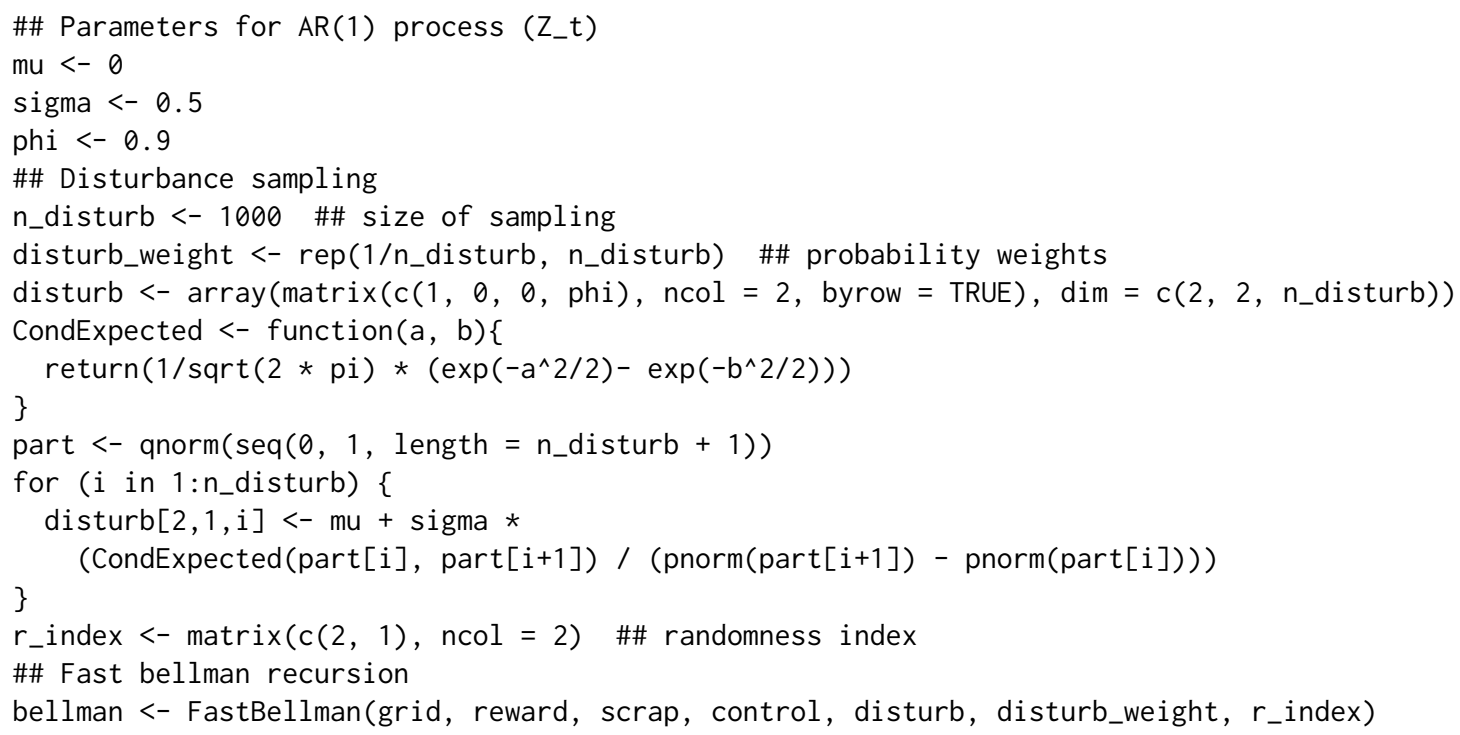

The following code example performs the solution diagnostics, computes the bound estimates, and then outputs the results (shown in Table 1). This gives the expected cumulative cost (and its $99 \%$ confidence interval) faced by the retailer depending on the current amount of stored energy in the battery. If interested, we point the reader to Hinz and Yee (2017a) for more details regarding this interesting problem.

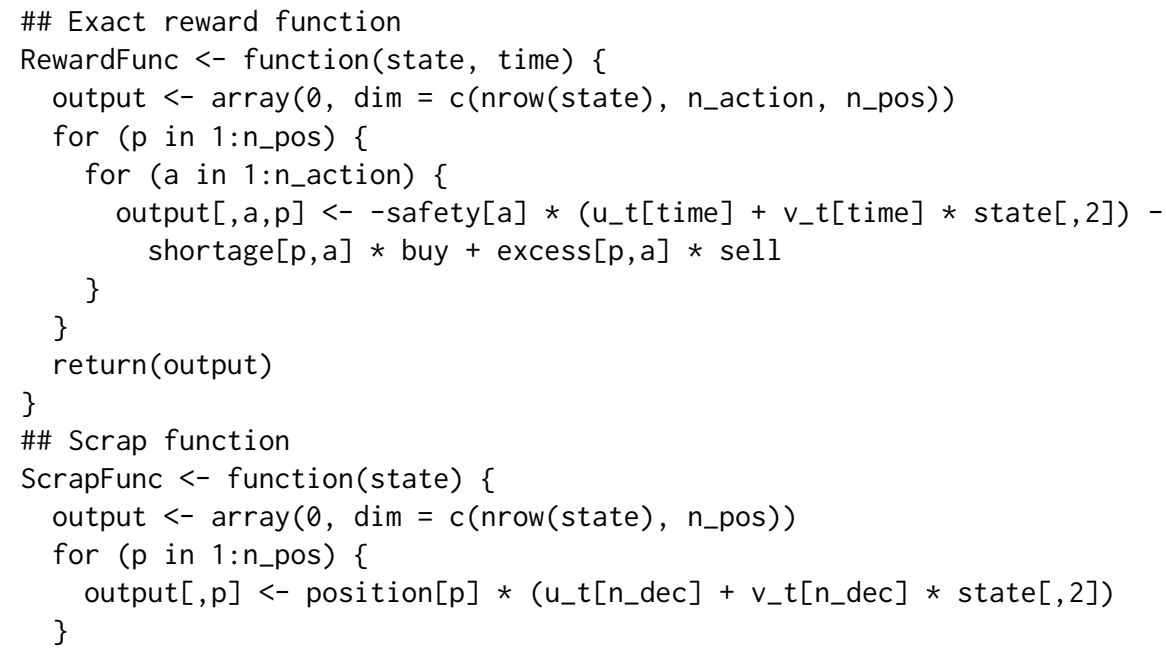




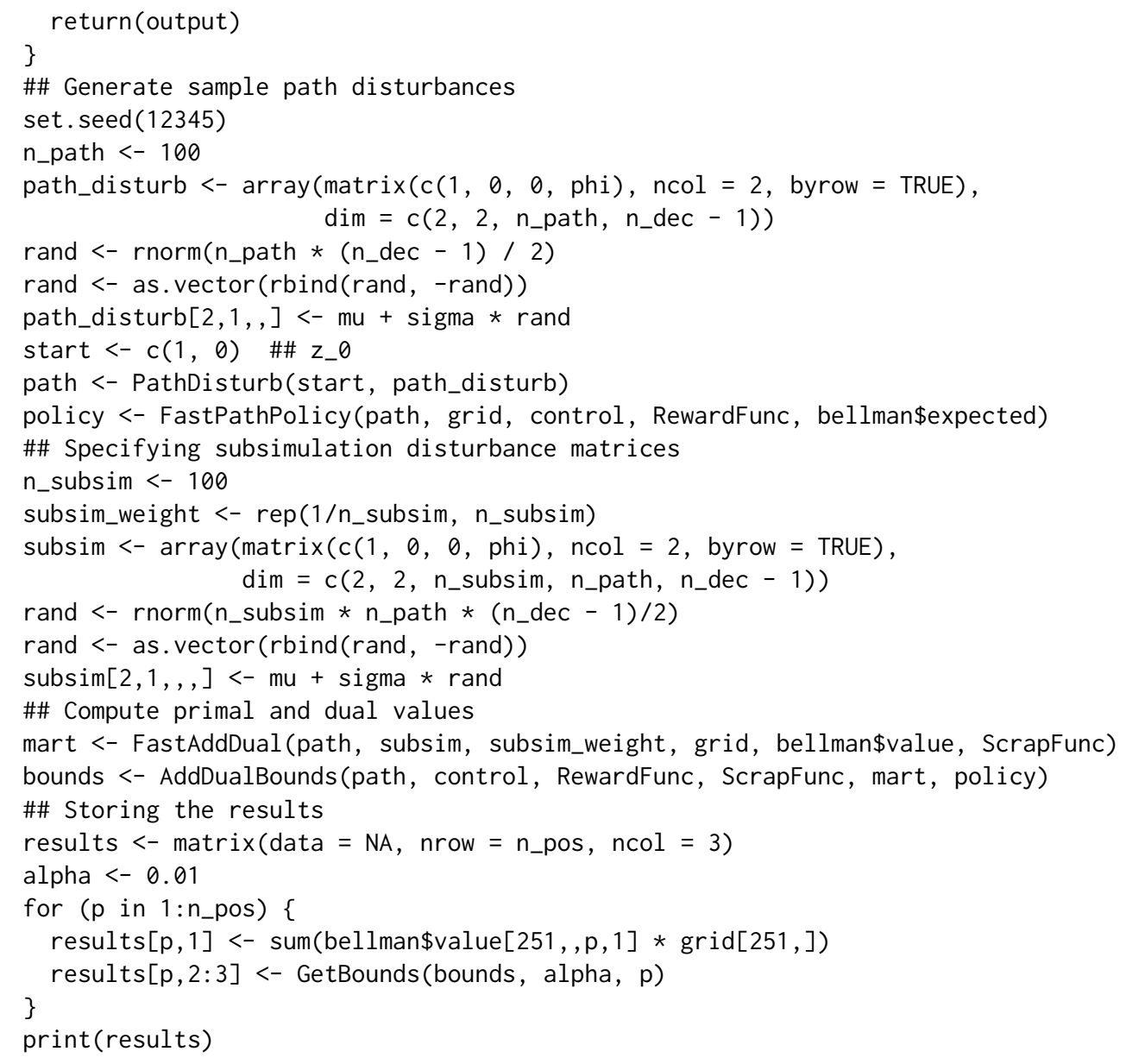

\begin{tabular}{ccc} 
Start Level (MWh) & Subgradient Estimate & $99 \%$ CI \\
\hline 0 & -1679.859 & $(-1679.862,-1679.612)$ \\
5 & -1629.859 & $(-1629.862,-1629.612)$ \\
10 & -1579.859 & $(-1579.862,-1579.612)$ \\
15 & -1529.859 & $(-1529.862,-1529.612)$ \\
20 & -1480.168 & $(-1480.171,-1479.922)$ \\
25 & -1433.574 & $(-1433.577,-1433.328)$ \\
30 & -1389.685 & $(-1389.688,-1389.441)$ \\
35 & -1348.509 & $(-1348.512,-1348.266)$ \\
40 & -1310.129 & $(-1310.131,-1309.887)$ \\
45 & -1274.601 & $(-1274.604,-1274.362)$ \\
50 & -1241.952 & $(-1241.955,-1241.714)$ \\
55 & -1212.186 & $(-1212.188,-1211.949)$ \\
60 & -1185.294 & $(-1185.297,-1185.059)$ \\
65 & -1161.261 & $(-1161.263,-1161.027)$ \\
70 & -1140.063 & $(-1140.066,-1139.831)$ \\
75 & -1121.677 & $(-1121.680,-1121.446)$ \\
80 & -1106.080 & $(-1106.083,-1105.850)$ \\
85 & -1093.250 & $(-1093.253,-1093.021)$ \\
90 & -1083.161 & $(-1083.164,-1082.932)$ \\
95 & -1075.727 & $(-1075.730,-1075.499)$ \\
100 & -1070.728 & $(-1070.731,-1070.500)$
\end{tabular}

Table 1: Subgradient and duality approach using $Z_{0}^{(2)}=0$. 


\section{Natural resource extraction}

As a final example, let us consider the case of optimal resource extraction in Hinz et al. (In Press). Suppose we have a hypothetical copper mine represented in Table 2.

\begin{tabular}{ll}
\hline Output rate: 10 million pounds/year & Inventory level: 150 million pounds \\
Costs of production: $\$ 0.50 /$ pound & Opening/closing costs: $\$ 200,000$ \\
Maintenance costs: $\$ 500,000 /$ year & Inflation rate: $8 \% /$ year \\
Convenience yield: $1 \% /$ year & Price variance: $8 \% /$ year \\
Real estate tax: $2 \% /$ year & Income tax: $50 \%$ \\
Royalty tax: $0 \%$ & Interest rate: $10 \% /$ year \\
\hline
\end{tabular}

Table 2: Hypothetical copper mine.

The following code approximates the value function of the above mine using the following setting:

- The mining lease is 30 years. After 30 years, the owner relinquishes ownership of the mine to the government.

- Time is discretized into quarter years.

- At the start of each time epoch, the owner decides whether the mine will be open (i.e. extracting ore) or closed (not extracting) for the next quarter. The owner can also relinquish ownership of the mine early at any of these time points.

- Switching between open and closed states incurs a switching cost.

- An open mine produces income but incurs higher operating costs than a closed mine.

- The amount of ore in the mine is finite and decreases whenever the mine is open.

- The owner wishes to maximise the expected total profits which gives the true value of the mine.

In the code below, the grid will be used to represent the price of the ore which is assumed to follow geometric Brownian motion. The control matrix will be used to reflect changes in the amount of unextracted ore based on the owner's dynamic decision to open or close. For the disturbance sampling, the disturbance space is partitioned into 1000 components of equal probability measure and the local averages on each partition component is taken.

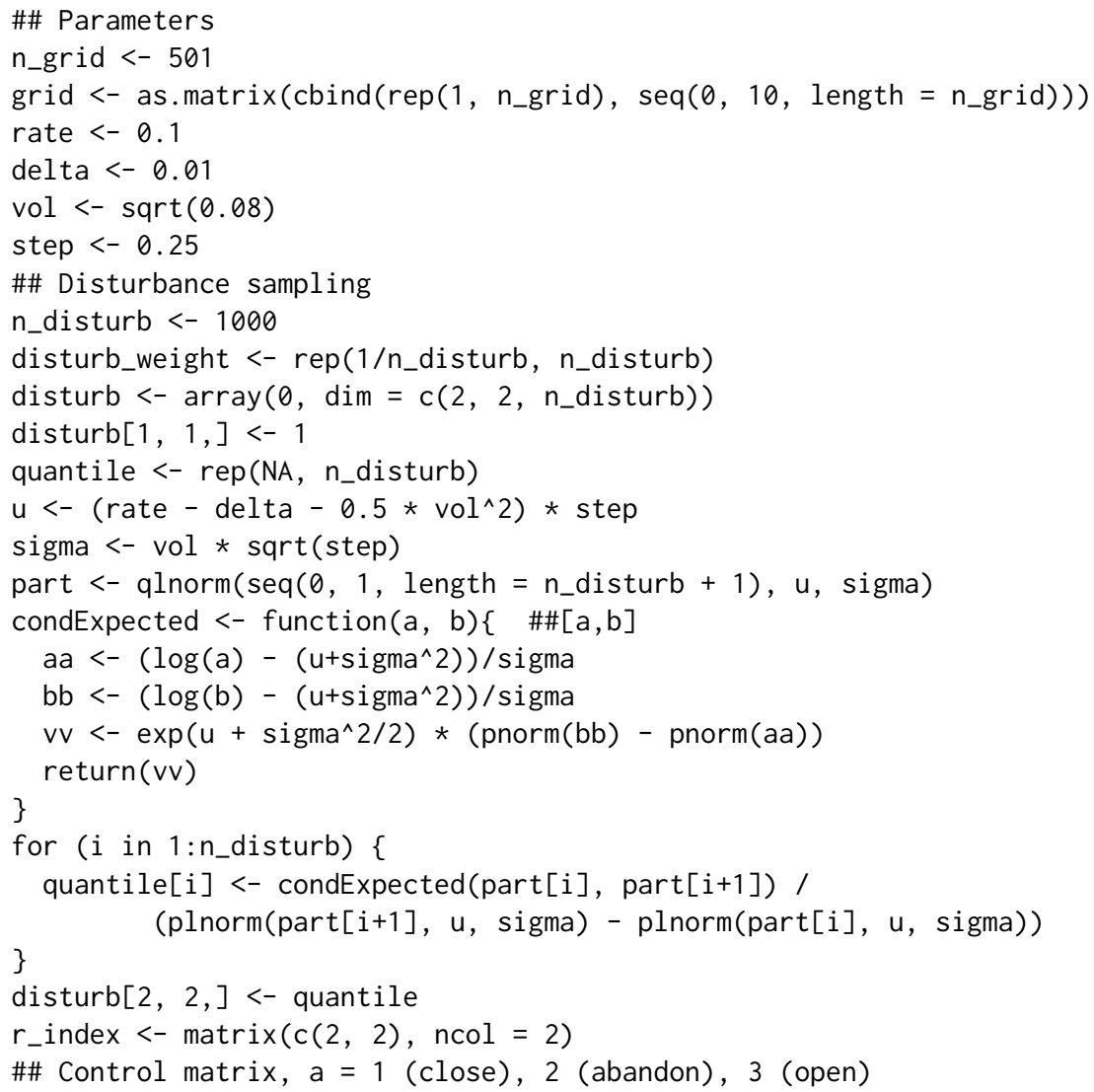


\#\# $p=1$ (exhausted), levels +1 (full open), 2 * levels +1 (full closed)

levels <- 15 / step

control <- matrix (data $=1$, nrow $=(2 *$ levels +1$)$, ncol = 3)

control[2: $(2 *$ levels +1$), 1]<-($ levels +2$):(2 *$ levels +1$)$

control[2: $(2 *$ levels +1$), 3]<-1:$ levels

Next, we define the subgradient representation of the reward and scrap functions. When the mine is open, the reward is essentially the income from selling the extracted ore minus the cost of extracting that ore. When the mine is closed, the reward is the cost of maintenance. If the owner switches between open and closed states, there is a switching cost. When the owner relinquishes ownership of the mine, there is no reward or cost. The scrap reward function is set to zero in the below code. These objects are then passed into the FastBellman function which computes the value function approximations using nearest neighbors.

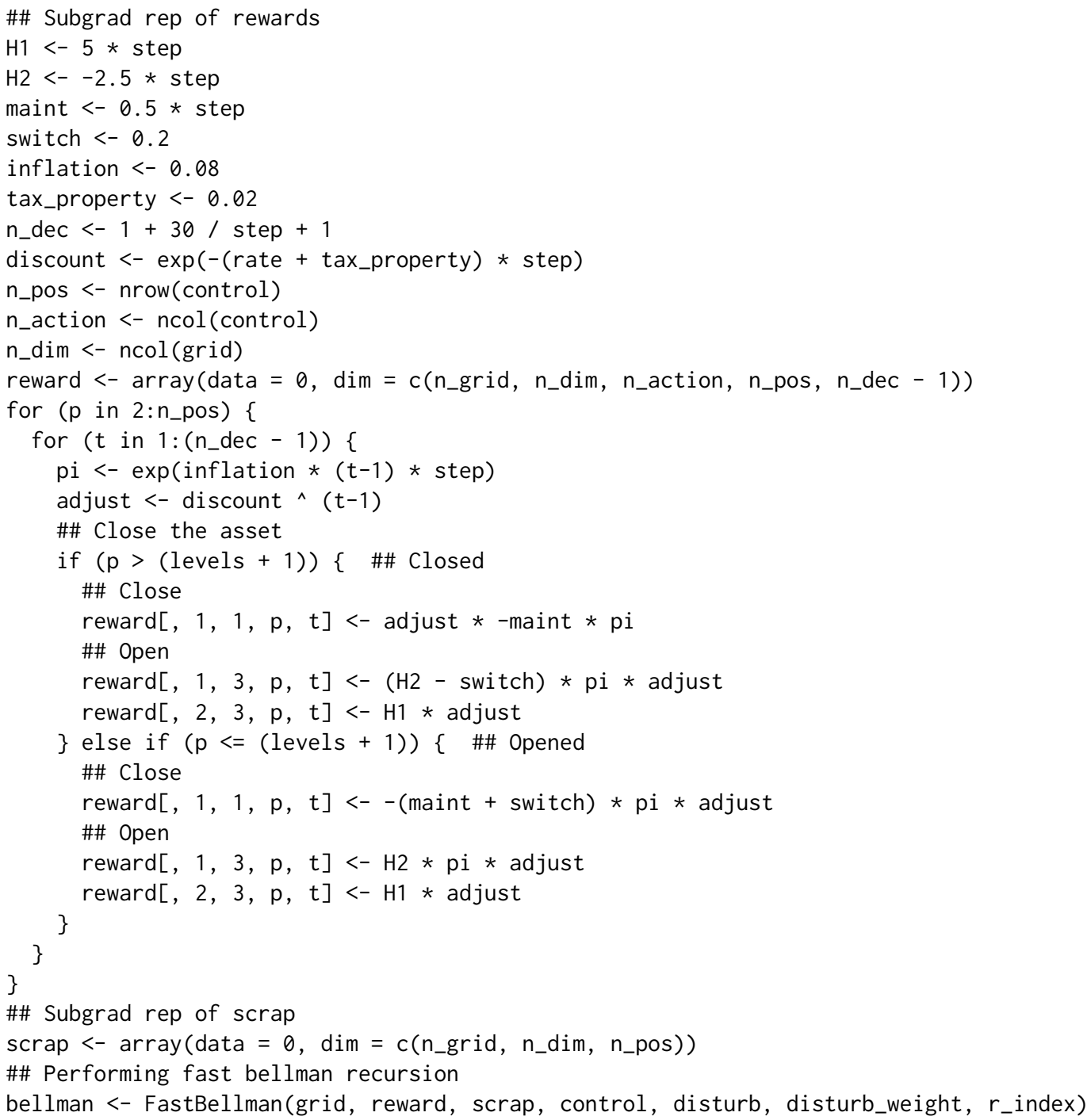

Finally, running the following code gives an illustration of the value of an open mine based on the current ore price.

plot(grid[,2], rowSums(bellman\$value[, levels,1] $*$ grid), type $=" 1 "$, $\mathrm{xlab}=$ "Current Ore Price $(\$) "$, ylab = "Mine Value (\$ millions)")

For more details regarding this problem, we point any interested readers to Hinz et al. (In Press).

\section{Conclusion}

This paper gives a demonstration of our R package, rcss in solving optimal switching problems. Our package represents the first software implementation of this class of algorithms. The problem setting 


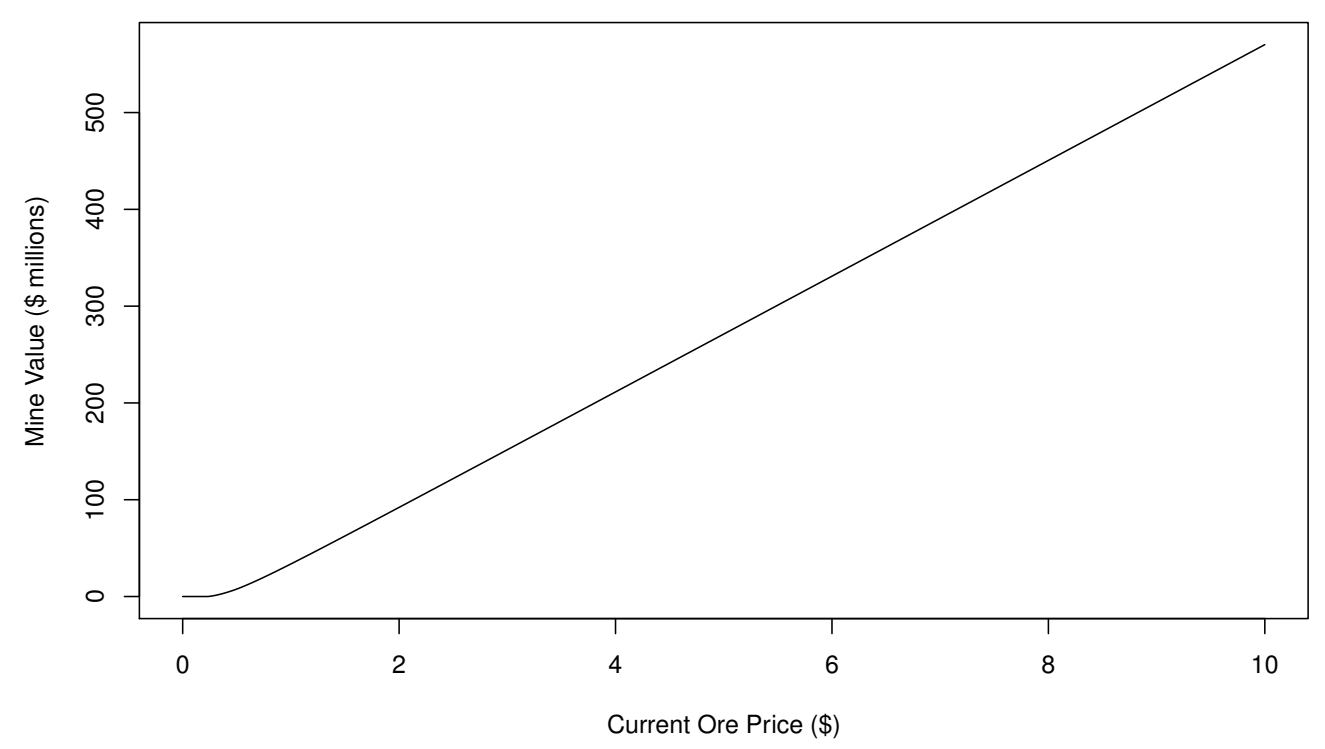

Figure 5: Mine valuation.

discussed in this paper is broad and can be used to model a wide range of problems. Using nearest neighbor algorithms, the package rcss is able to solve some real world problems in an accurate and quick manner. It is also worth mentioning that this $\mathrm{R}$ package can be used to address Markov decision processes when there is a hidden Markov model as shown in Hinz and Yee (2017b). However, this is not demonstrated here in order to preserve the brevity of this paper.

\section{Bibliography}

N. Bauerle and U. Rieder. Markov Decision Processes with Applications to Finance. Springer-Verlag, 2011. URL https://doi.org/10.1007/978-3-642-18324-9. [p38]

L. Dagum and R. Menon. Openmp: An industry standard api for shared-memory programming. IEEE Computational Science E Engineering, 5(1):46-55, 1998. [p40]

D. Eddelbuettel and R. Francois. Rcpp: Seamless R and C++ integration. Journal of Statistical Software, 40(8):1-18, 2011. URL https://doi .org/10.18637/jss.v040.i08. [p40]

P. Glasserman. Monte Carlo Methods in Financial Engineering. Springer-Verlag, 2003. [p40]

J. Hinz. Optimal stochastic switching under convexity assumptions. SIAM Journal on Control and Optimization, 52(1):164-188, 2014. URL https://doi.org/10.1137/13091333x. [p38, 40]

J. Hinz and N. Yap. Algorithms for optimal control of stochastic switching systems. Theory of Probability and its Applications, 60(4):770-800, 2015. [p38, 40]

J. Hinz and J. Yee. Optimal forward trading and battery control under renewable electricity generation. Journal of Banking E Finance, InPress, 2017a. ISSN 0378-4266. URL https://doi .org/10.1016/j . jbankfin. 2017.06.006. [p38, 47,49]

J. Hinz and J. Yee. Stochastic switching for partially observable dynamics and optimal asset allocation. International Journal of Control, 90(3):553-565, 2017b. [p38,53]

J. Hinz, T. Tarnopolskaya, and J. Yee. Efficient algorithms of pathwise dynamic programming for decision optimization in mining operationsh. Annals of Operations Research, In Press. [p38, 51, 52]

S. Mangenat and G. Jefferies. Nabor: Wraps 'Libnabo', a Fast K Nearest Neighbour Library for Low Dimensions, 2018. URL https: //CRAN. R-project. org/package=nabor. R package version 0.5. [p40]

H. Pham. Continuous-Time Stochastic Control and Optimization with Financial Applications, volume 61. Springer-Verlag, 2009. [p38] 
W. Powell. Approximate Dynamic Programming: Solving the Curses of Dimensionality. John Wiley \& Sons, 2007. URL https://doi.org/10.1002/9781118029176. [p38]

R Core Team. R: A Language and Environment for Statistical Computing. R Foundation for Statistical Computing, 2013. URL http: //www. R-project.org/. ISBN 3-900051-07-0. [p38]

J. Yee. Convex function approximations for Markov decision processes. arXiv:1712.00970, Preprint. [p38]

Juri Hinz

University of Technology Sydney

Juri.Hinz@uts.edu.au

Jeremy Yee*

*Corresponding Author

University of Technology Sydney

jeremyyee@outlook.com.au 